\title{
Contando y Capturando Cocodrílidos ${ }^{1}$
}

\author{
Michael S. Cherkiss, Holly E. Fling, Frank J. Mazzotti, Kenneth G. Rice y Marianna D. Conill ${ }^{2}$

\section{Introducción}

\section{Encontrando Cocodrílidos}

Con pocas excepciones, los cocodrílidos deben ser contados y capturados para estudiar su ecología. La mayoría de los métodos utilizados hoy en día para encontrar y capturar cocodrílidos son modificaciones de los métodos utilizados para su caza (Chabreck 1963; Jones 1966). Estos métodos han sido modificados según la especie en estudio o según las condiciones de su hábitat específico, así como para minimizar el riesgo de daño que puede ser causado tanto a los cocodrilos como al investigador (Webb y Messel 1977; Hutton et al. 1987; Walsh 1987; McDaniel y Hord 1990).

Cualquier situación con demandas especiales, requerirá de innovaciones en los métodos utilizados.

\section{Medios de Transporte}

Los cocodrilos deben ser ubicados antes de poder ser contados o capturados. Varias técnicas son utilizadas dependiendo del hábitat, costo, logística de campo y metas del estudio. Ocasionalmente se puede correr con la suerte de poder acercarse a un cocodrilo en automóvil, y a veces los cocodrílidos pueden encontrarse haciendo su búsqueda en caminatas. Como los cocodrílidos viven en ambientes acuáticos, usualmente se requiere del uso de un medio de transporte acuático. Se pueden utilizar avionetas y helicópteros cuando las áreas no son accesibles en bote, y también para cubrir mayores áreas en un corto período de tiempo. Los trabajos diseñados para obtener información detallada del uso del hábitat y de la demografía normalmente son realizados utilizando botes.

Los medios de transporte acuático utilizados para encontrar cocodrílidos usualmente tienen un casco plano con un interior abierto, y están hechos

1. Este documento es la Circular 1451S, una de la serie del Wildlife Ecology and Conservation Department, Florida Cooperative Extension Service, Institute of Food and Agricultural Sciences, University of Florida. Publicado por primera vez: Diciembre, 2005. Por favor visite la página web EDIS http://edis.ifas.ufl.edu. La inclusión de marcas específicas dentro de este artículo no constituye la aprobación de estos productos en preferencia de otros. La mención de un producto patentado no constituye una garantía de los productos por los autores o la editorial.

2. Michael S. Cherkiss, Ecólogo de Fauna Silvestre; Holly E. Fling, Asistente de Investigación de Fauna Silvestre; Frank J. Mazzotti, Profesor Asociado; Marianna Domínguez Conill, Asistente de Investigación de Fauna Silvestre; Wildlife Ecology and Conservation Department, University of Florida, Fort Lauderdale Research and Education Center, Florida 33314, Florida Cooperative Extension Service, Institute of Food and Agricultural Sciences, University of Florida, Gainesville. Kenneth G. Rice, Biólogo de Investigación de Fauna Silvestre, USGS Center for Water and Restoration Studies, Fort Lauderdale Research and Education Center, Florida 33314.

EI Instituto de Alimentos y Ciencias Agrícolas es un empleador que opera bajo Acción Afirmativa y provee Oportunidades Igualitarias, autorizado a proveer investigación, información educativa y otros servicios, únicamente a los individuos e instituciones que operan sin discriminación alguna con relación al credo, color, religión, edad, incapacidad, sexo, orientación sexual, estado civil, nacionalidad, opinion política o afiliaciones. Para más información sobre como obtener otras publicaciones de extensión, comuníquese con la oficina de Servicio de Extensión de su condado. Servicio de Extensión de la Florida / Instituto de Alimentos y Ciencias Agrícolas / Universidad de la Florida / Larry Arrington, Decano. 
de alguna combinación entre fibra de vidrio, madera, aluminio y plástico. En hábitats acuáticos se pueden utilizar kayaks, canoas, botes de aluminio, piraguas, botes de aire, lanchas de pesca y de pantano, entre otros; para atravesar grandes expansiones de áreas secas entre zonas acuáticas, se suele utilizar transporte anfibio todo-terreno (Figura 1). fuera de borda más grandes, los cuales pueden o no estar permanentemente adheridos al bote. Los motores fuera de borda tradicionales son eficientes en aguas abiertas, y en áreas con densa vegetación, lodo, tocones y rocas, se aconsejan los motores que ofrecen los Fabricantes Go-Devil de Louisiana, Inc. (www.go-devil.com). Los botes de pesca también pueden ser desplazados mediante el uso de varas o

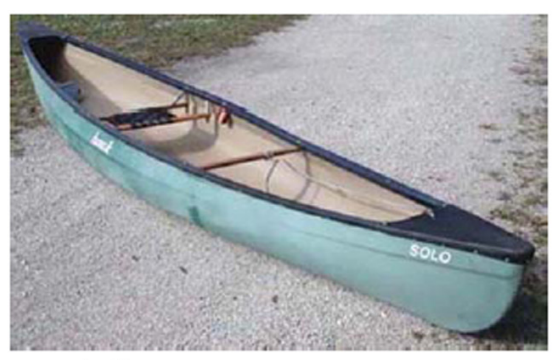

A. Canoa

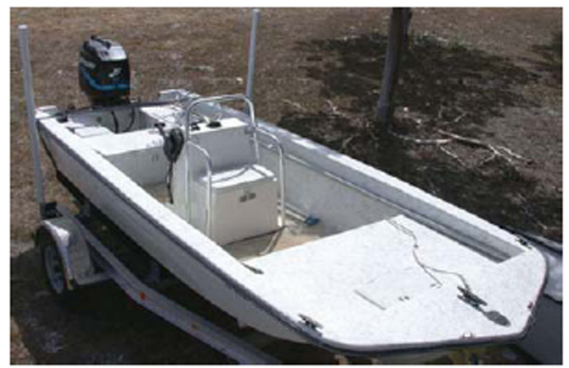

C. Lancha de Pantano

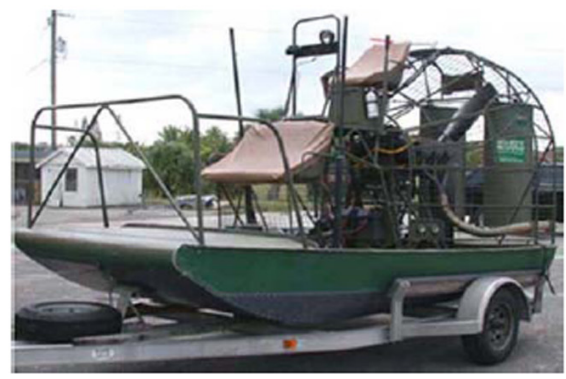

E. Bote de Aire

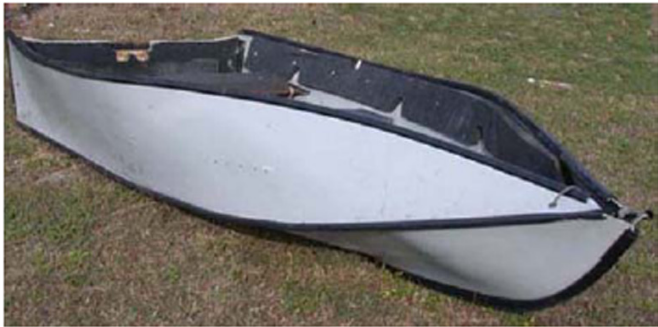

B. Bote Transportable

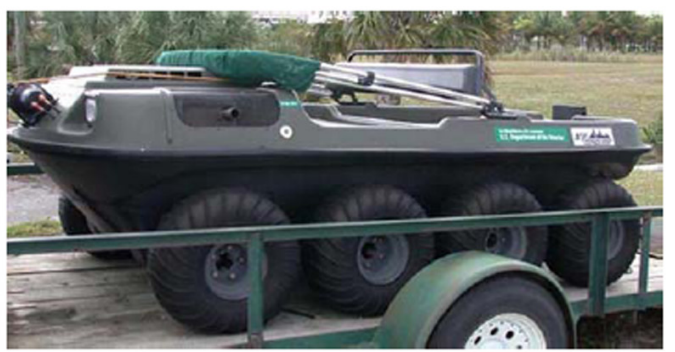

D. Vehiculo Todo-terreno

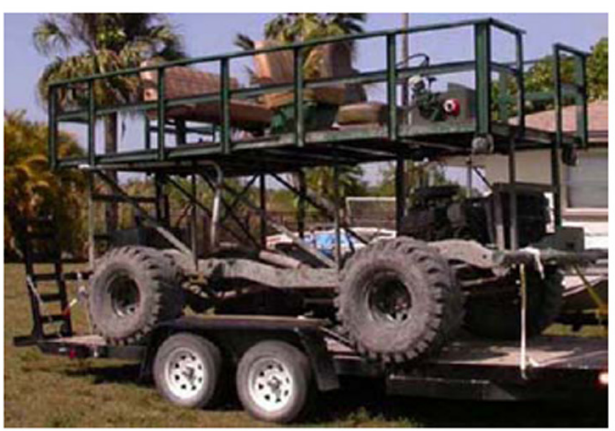

F. Buggy de Pantano

Figura 1. Distintos medios de transporte utilizados para la captura de cocodrílidos y estudios de investigación. Créditos fotográficos: Michael Cherkiss.

Los métodos de propulsión utilizados son tan variados como las embarcaciones. Los kayaks, botes de aluminio, piraguas, y canoas pueden ser propulsados mediante remos, varas o palos de empuje, pequeños motores portátiles fuera de borda, motores internos y motores eléctricos. Las lanchas de pesca son propulsadas mediante motores para palos de empuje en áreas demasiado llanas para el uso de motores. Los botes de aire presentan un casco de fondo plano y son fabricados de aluminio o fibra de vidrio. Estos botes de aire contienen una propela, similar a las hélices de un avión, la cual se encuentra montada en una jaula en la popa del bote. Los botes de aire pueden funcionar con motores de 
automóvil o de naves aéreas. Existen botes de aire que están diseñados especialmente para funcionar sobre aguas someras o en ausencia completa de agua. En estos botes de "pantano", los motores de naves aéreas son más efectivos ya que deben tener una mejor relación caballos de fuerza/peso.

El uso de vehículos todo-terreno es necesario cuando los humedales estacionales se encuentran en época de sequía, obligando a los cocodrílidos a concentrarse en charcos y lagunas aisladas. En los Llanos de Venezuela se pueden utilizar vehículos todo terreno $4 \mathrm{x} 4$ para visitar los pozos de caimanes, pero los investigadores generalmente van a caballo. En la Florida existe una gran variedad de "vehículos de pantano", construidos especialmente para suelos sumamente suaves o rocosos, o para aguas profundas. El vehículo anfibio todo-terreno mostrado en la Figura 1 puede ser utilizado como un vehículo con ruedas o con bandas de rodamiento (semejantes a las de los tanques) y es capaz de atravesar aguas profundas, tales como canales. Con las bandas de rodamiento, este vehículo tiene una presión de impacto sobre el suelo de menos de $60 \mathrm{~g}$ por centímetro cuadrado. Esto representa una presión menor a la generada por una pisada de un ser humano y minimiza el daño en medios ambientes sensitivos.

\section{Navegación}

Para localizar y estudiar el área de interés se deben tener habilidades en el uso de mapas y compás y, después de localizar a un cocodrilo, es necesario encontrar el camino de regreso al punto de partida. Los sitios de visión y/o captura pueden ser marcados en un mapa bien detallado, pero siempre hay cabida para errores humanos. Los recibidores de Sistemas de Posicionamiento Global (GPS, por sus siglas en inglés) son de gran ayuda ya que las localidades pueden ser grabadas. Los recibidores GPS pueden proveer mapas del área (utilizando el software apropiado), mostrar caminos, grabar localidades de visiones o captura, grabar datos del medio ambiente u otras características, a la resolución apropiada para la unidad utilizada en los análisis posteriores y asistir en la navegación, pero no pueden reemplazar las habilidades básicas de orientación. El uso de Asistentes Digitales
Personales (PDA, por sus siglas en inglés) también se está popularizando para el almacenamiento de datos (Waddle et al. 2003).

\section{Luces}

La mejor hora para contar o capturar cocodrílidos es durante la noche. Esto se debe a que estos animales son más activos durante la noche y sus candiles (luz reflejada del tapetum lucidum) los hacen visibles. Por estas razones los estudios de candiles de noche o de lámparas encandiladoras son los métodos más utilizados para encontrar cocodrilos (Magnusson 1982). Una gran diferencia viene dada por el tipo de luz. Las bombillas de halógeno y xenón producen una luz bastante brillante. Las luces marinas y de buceo también son buenas fuentes de luz para investigadores de cocodrilos, ya que pueden soportar las condiciones de humedad bajo las cuales estos estudios normalmente se conducen. Las luces brillantes no siempre son las mejores; luces demasiado brillantes pueden eliminar los candiles de pequeños cocodrilos así como los de cocodrilos que se encuentren más cercanos. Las luces en el rango comprendido entre $50.000 \mathrm{y}$ 200.000 candelas son más efectivas y los haces azules son menos brillantes que los blancos. Cuando no se disponga de un proyector de luz orientable, estos pueden fabricarse a partir de una batería y un faro delantero de automóvil.

Las linternas de cabeza son tan importantes para capturar cocodrilos como los proyectores de luz. Éstas son esenciales cuando el medio de transporte es manejado manualmente, así como durante los eventos de captura donde las manos deben estar libres. Existen distintos tipos de linternas de cabeza que han sido diseñadas para la caza, minería y para acampar.

\section{Contando Cocodrílidos}

\section{Informantes}

Para obtener información acerca de los antecedentes del área a visitar y de las necesidades actuales de investigación en ésta, utilizar datos previos puede ser de gran ayuda así como entrevistar directamente a los integrantes de las comunidades locales (Thorbjarnarson 1988; Thorbjarnarson y 
Hernández 1988). Desafortunadamente, las comunicaciones personales suelen ser sesgadas, irreplicables y la información puede estar desactualizada o ser inespecífica. A pesar de los defectos como técnicas de investigación, las entrevistas pueden ayudar a identificar las áreas apropiadas de estudio, pueden proveer información que de alguna otra manera no podría ser obtenida y ayudan a desarrollar importantes vínculos con la comunidad local.

\section{Artefactos}

Los artefactos o rastros más comunes dejados por cocodrílidos son huellas, nidos y heces. La búsqueda de señales de cocodrílidos puede ser útil para determinar la presencia, mas no ausencia, de un animal. Las huellas en el sustrato pueden ser generadas por el reptar o el arrastre de la cola. Los nidos y los sitios de anidación pueden ser localizados explorando la línea de la costa desde un bote, un bote de aire, o simplemente caminando a lo largo de canales y riachuelos. Los estudios aéreos también pueden ser llevados a cabo para localizar nidos (Magnusson et al. 1980; McNease et al. 1994; Brandt et al. 1995). Una cuarta señal que indica la presencia de cocodrilos es auditiva, ya que algunos cocodrilos rugen en época de apareo o cuando se sienten amenazados. La combinación de los métodos mencionados permite una cobertura más completa del área de estudio (Magnusson et al. 1978) (Figura 2).

\section{Estudios}

\section{Conteos Terrestres Diurnos}

Este tipo de conteo es el más fácil de programar y además es el método que requiere menos equipo. Sin embargo, muchos conteos donde no se ha encontrado ningún animal han sido asociados al Conteo Terrestre Diurno, ya que los cocodrílidos suelen abstenerse de asolearse cuando el tiempo se encuentra nublado o cuando ya han alcanzado la temperatura corporal óptima (O'Brien 1990). Además, a finales de primavera la actividad diurna comienza a disminuir aumentando a su vez la actividad nocturna, lo que hace que los conteos nocturnos sean los más apropiados. Este método de conteo se puede realizar a pié o desde un vehículo

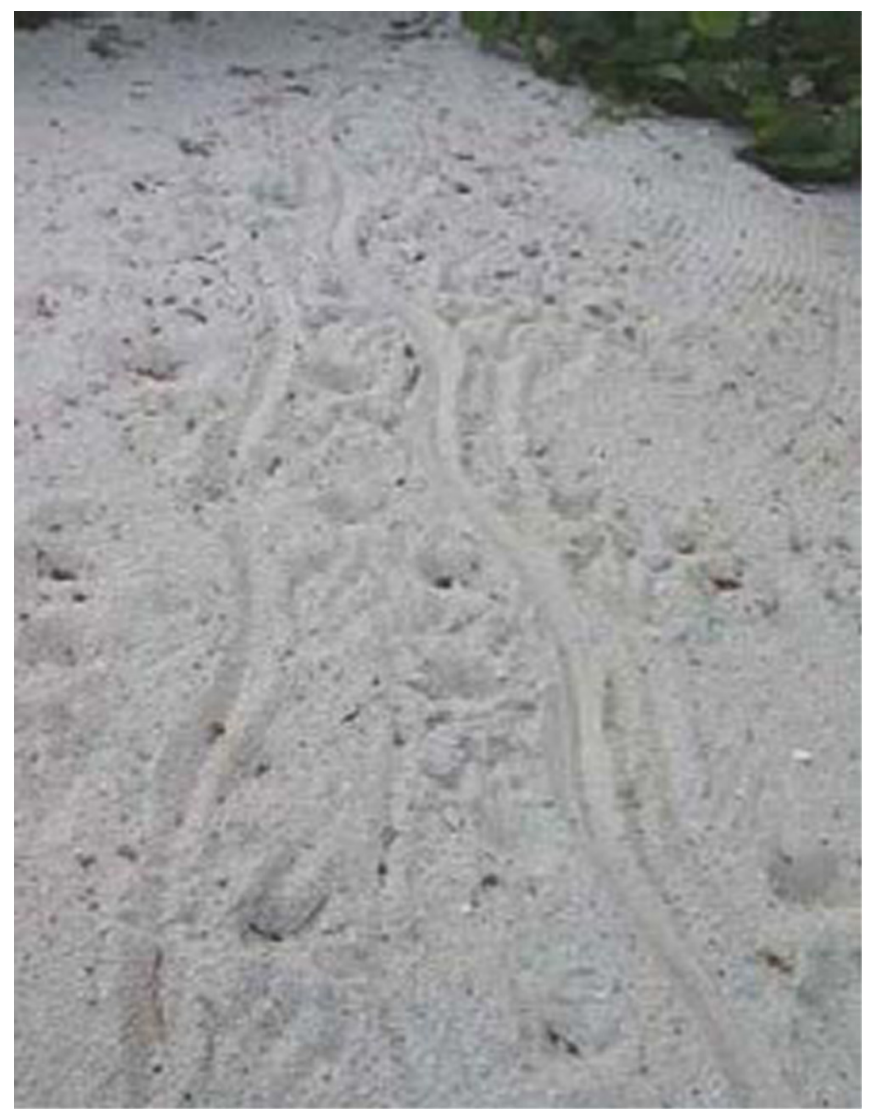

Figura 2. Huella dejada por el arrastre de la cola de un cocodrilo en una playa arenosa. Créditos fotográficos: Michael Cherkiss.

terrestre lo que puede ayudar a reducir los costos logísticos y de transporte (Magnusson 1982).

\section{Conteos Aéreos Diurnos}

El método de conteo aéreo diurno ha sido ampliamente aceptado como un método costo-efectivo para estudiar el hábitat de cocodrílidos gracias al amplia área que se puede cubrir en un período de tiempo limitado (Magnusson 1982; O'Brien 990). Utilizar transporte aéreo es muy útil en hábitats donde no se pueden navegar botes de propela o donde los botes de aire encuentran dificultad para desplazarse. Los helicópteros o avionetas deben volar entre los 50 y $80 \mathrm{~m}$ de altura sobre la tierra para obtener un conteo adecuado.

Se han obtenido conteos exitosos volando en helicópteros a una velocidad de 80-100 Km/hr y en avionetas a $120 \mathrm{Km} / \mathrm{hr}$ (Kushlan and Mazzotti 1989). Los Conteos Aéreos Diurnos han demostrado ser más efectivos en hábitats de pantanos abiertos y de 
lagos para detectar la distribución y abundancia de cocodrílidos o nidos, y menos efectivos en áreas donde existe un dosel cerrado que bloquea la visibilidad (Magnusson 1982; OBrien 1990).

\section{Conteos Nocturnos con Lámparas Encandiladoras}

Los conteos nocturnos representan el método más utilizado para los estudios de cocodrílidos (Magnusson 1982) gracias a su versatilidad en hábitat y a la naturaleza nocturna de estos animales. Este método se suele realizar desde un medio de transporte acuático, pero también se puede desarrollar desde tierra, utilizando un medio de transporte terrestre o caminando. Los candiles permiten al investigador visualizar al animal a largas distancias y en situaciones donde el animal no hubiese sido visto dadas otras circunstancias (OBrien 1990). Este método requiere que los ojos del animal se encuentren sobre la superficie del agua y su sumergimiento puede prevenir que sea visualizado. Además, se pueden obtener datos inconsistentes como consecuencia de errores humanos, de diferencias en el tipo de bote o de luz utilizada, así como de las condiciones ambientales (Pacheco 1996). Este método puede representar el esfuerzo terrestre más costoso, pero se obtienen los datos poblacionales más confiables. Esta técnica también permite colectar datos ambientales y detalles específicos del animal y su condición.

\section{Limitaciones}

Sin tomar en cuenta la densidad poblacional, existen otros factores que pueden afectar el número de cocodrílidos observados durante un conteo y es normal que no todos los cocodrílidos en un área sean contados (Graham y Bell 1969; Magnusson et al. 1978; Bayliss 1987). Las condiciones ambientales, tales como viento, temperatura del agua, temperatura ambiental y oleaje, afectan el conteo de cocodrílidos (Woodward y Marion 1979). La accesibilidad y el tipo de hábitat (p.ej.: lago o pantano) también afectan la proporción de cocodrílidos observados, así como la habilidad del observador, y los tipos de bote, motor (o ausencia del mismo) y luz utilizados. Dos observadores utilizando lámparas independientemente, pueden estimar cocodrílidos potencialmente visibles pasados por alto (cuando un observador ve un cocodrilo que el otro observador no vió) en un conteo (Magnusson et al. 1978; Bayliss 1987). Esto no toma en cuenta los cocodrílidos que no fueron observados por encontrarse sumergidos (animales cautelosos u otras razones) o que fueron opacados por su posición, la vegetación, o alguna otra característica del hábitat. La cautela de los cocodrilos puede ser estimada por la distancia a la que un observador se puede acercar a un cocodrilo antes de que éste se sumerja (Webb y Messel 1979; Pacheco 1996). La visibilidad en distintos hábitats puede ser probada explorando el área de muestreo detalladamente al día siguiente de un muestreo, tomando ventaja de la luz del día, o haciendo que un equipo coloque cocodrílidos falsos a ser contados por otro equipo distinto.

\section{Capturando Cocodrílidos}

Uno de los retos en la captura y manipulación de cocodrílidos es su extraordinario rango en tamaños. Los juveniles, de alrededor de $25 \mathrm{~cm}$ de largo y $25 \mathrm{~g}$ de peso, suelen ser inocentes y fáciles de agarrar con las manos. Es difícil acercarse a los cautelosos adultos, que pueden llegar a medir hasta 5 $\mathrm{m}$ y pesar $500 \mathrm{Kg}$. Los cocodrílidos capturados deben ser restringidos, muestreados, marcados y liberados ilesos. En la manipulación de grandes cocodrilos, soltar las ataduras y liberar al animal son los aspectos más peligrosos de una tarea bien arriesgada por sí sola.

El uso de trampas y la caza son las maneras básicas para capturar cocodrílidos. La decisión entre el uso de la caza o de trampas, y de la técnica a utilizar va a depender de muchos factores: tamaño, cautela, tipo de piel o cuero de la especie blanco, hábitat en el que se encuentra, tipo de transporte utilizado para encontrarlos, y el destino del animal después de la captura. Se pueden emplear métodos que causen heridas mortales al animal sólo en el caso de que se planee aniquilar al cocodrílido después de su captura. Como la mayoría de los estudios ecológicos involucran animales vivos, aquí se describirán métodos que están diseñados para la seguridad tanto del cocodrílido como del investigador, con algunas excepciones (p.ej. anzuelos con carnada y arpones). 


\section{Trampas}

La ventaja que poseen las trampas es que se pueden capturar animales que son muy cautelosos a los cuales no lograría siquiera acercárseles un humano. Sin embargo, las trampas no son selectivas. Se pueden atrapar animales de todos los sexos y tamaños. Además, hasta que no se chequee la trampa, un cocodrilo atrapado corre el riesgo de herirse por forcejeo, ahogo, recalentamiento o ataque (Walsh 1987; Leslie 1997). Por esta razón es muy importante chequear las trampas a intervalos regulares. También se pueden instalar transmisores para que emitan una señal cuando la trampa sea activada (Webb y Messel 1977). terrestres o acuáticas para la captura de los animales. Si esto no es exitoso, quizás se necesite colocar cebo para atraer a un cocodrílido a la trampa. Una ventaja del uso de cebo, es que puede ser atado a un dispositivo que acciona acelerando el cierre de la trampa (Murphy y Fendley 1975; Leslie 1997). Para accionar o soltar las trampas se ha utilizado contrapeso, bambú, varas de fibra de vidrio, resortes planos y tubería de caucho (Pitman 1941; Murphy y Fendley 1975; Webb y Messel 1977; Hutton et al. 1987; Walsh 1987). Una desventaja del uso de cebo es que otros animales pueden comerla primero. De esta manera, la trampa se queda sin cebo, se puede

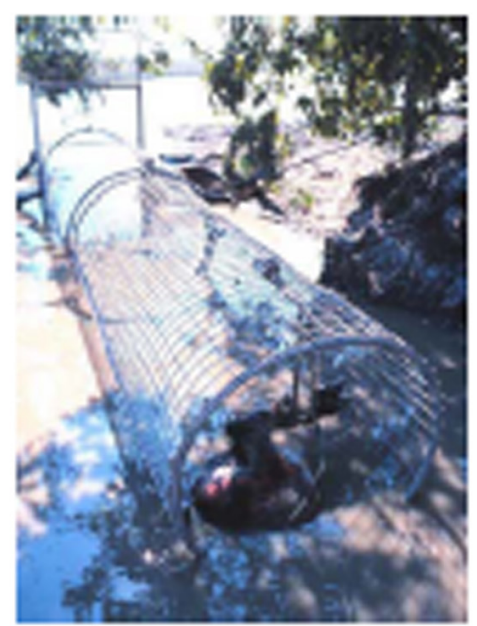

A. Trampa Cilindrica en Tierra

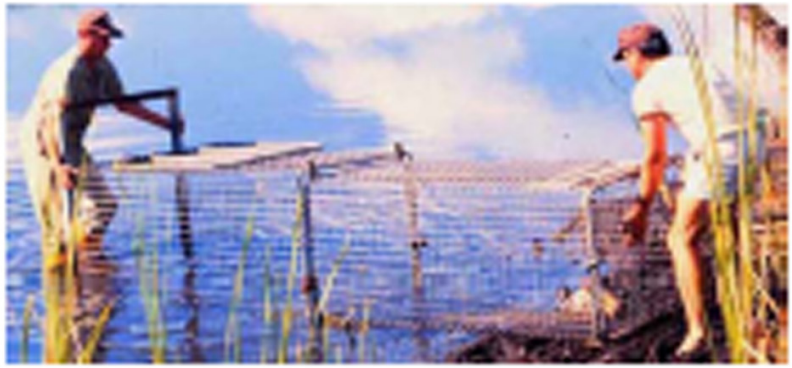

B. Trampa de Caja

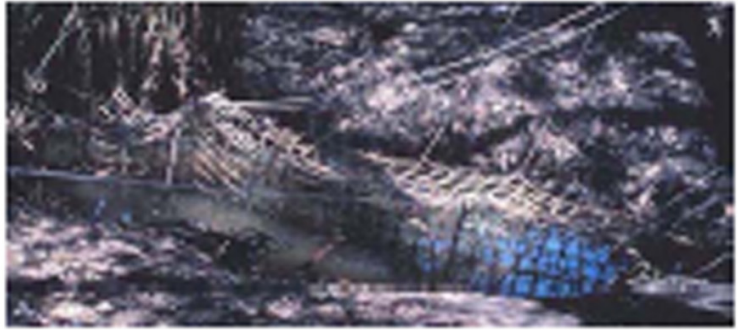

C. Trampa de Red

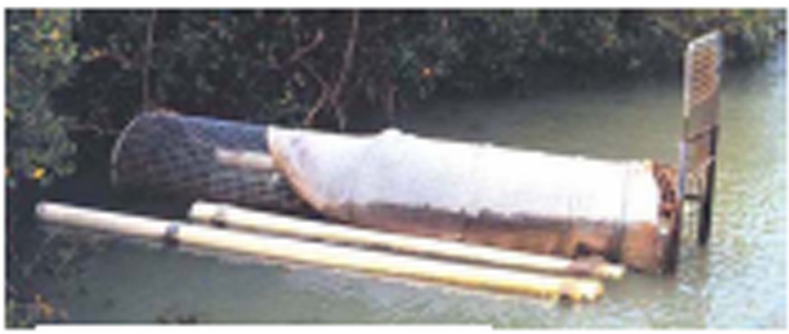

D. Trampa Cilindrica en Agua

Figura 3. Distintos tipos de jaulas y trampas de red utilizadas en la captura de cocodrílidos. Créditos fotográficos: Graham Webb.

Los hábitats de uso regular, como caminos, sitios de anidación, sitios para asolearse o madrigueras, son buenos sitios para colocar trampas activar o inhabilitar el dispositivo que acciona, y hasta se puede atrapar al animal equivocado. 
Para la captura de cocodrílidos que van a ser aniquilados resulta efectivo el uso de anzuelos grandes provistos de cebo y colocados sobre un cuerpo de agua. Para cocodrílidos que van a ser liberados, se pueden utilizar anzuelos digeribles cebados. Los anzuelos o ganchos digeribles pueden ser hechos pasando una soga de fibra natural (algodón o cáñamo) a través de un hueso hueco de ave (Walsh 1987) o de una espiga de madera, y envolviendo este dispositivo con un caparazón de pollo, o con pulmones de cerdo o vaca. Una vez que el cebo ha sido tragado, la espiga o el hueso se fija dentro del estómago "enganchando" efectivamente al cocodrílido. La soga puede cortarse después de que el animal ha sido recuperado, y el aparato que se encuentra en el estómago será digerido. Un lazo corredizo cebado puede ser utilizado para capturar al cocodrilo por una mandíbula. Esta técnica aumenta el riesgo de lesión en las trampas terrestres o acuáticas, pero sirve muy bien para animales de mandíbulas pesadas (Kofron 1989). Al utilizar dispositivos que accionen, trampas cebadas, lazos corredizos, redes, cajas o cilindros, es de suma importancia asegurarlos apropiadamente. Las trampas de caja o cilíndricas son difíciles de manejar, los cocodrílidos eventualmente le cogen miedo a estas trampas (Leslie 1997) y se pueden lastimar fácilmente al forcejear por liberarse (Walsh 1987). Los lazos corredizos y las redes son efectivas para grandes cocodrílidos, causan lesión mínima y son reutilizables (Pitman 1941; Murphy y Fendley 1975; Webb y Messel 1977; McDaniel y Hord 1990; Leslie 1997) (Figura 3).

Las trampas terrestres se pueden colocar en caminos (Mazzotti y Brandt 1988; Wilkinson 1994) (Figura 4), y las redes flotantes pueden ser colocadas a lo largo de pozos o riachuelos (Webb y Messel 1977). Las redes flotantes también capturan peces, los cuales pueden servir como cebo. Las redes deben ser supervisadas casi continuamente para prevenir que cocodrílidos u otros animales sean capturados y corran el riesgo de ahogarse. Los cocodrílidos en guaridas o cavernas pueden ser capturados colocando lazos corredizos o redes en las salidas de estos lugares. A veces el rastro dejado por un cocodrílido puede seguirse en la superficie de la tierra sondando en el suelo con una barra de metal. El investigador puede entonces incitar al animal a salir de la guarida punzándolo. El investigador también puede cavar hacia la guarida, de esta manera exponiendo al cocodrilo para la captura. Nosotros no recomendamos cavar las guaridas, ya que esto destruye una característica crítica del hábitat de la que pueden depender otros animales acuáticos.

Una trampa de asoleamiento es una trampa de tortuga modificada (Petokas y Alexander 1979) y es efectiva para animales pequeños y cautelosos. Se coloca una plataforma flotante donde se han avistado pequeños cocodrilos tomando sol, y se rodea de redes. Un cocodrilo asustado se lanzará fuera de la plataforma hacia las redes. Si es posible, el remover sitios de asoleamiento alternativos incitará a que los cocodrilos utilicen la plataforma para asolearse.

El uso de trampas de cámara no involucra la manipulación de animales (Thorbjarnarson et al. 2000). Las cámaras ópticas o digitales son colocadas en áreas de actividad (los sitios de anidación son particularmente productivos) y se activan por sensores de calor o de movimiento. La fotografía es estampada con la fecha y hora del evento. La cantidad y tipo de datos colectados se ven limitados por la presencia y comportamiento de los animales ya que éstos no son manipulados directamente por el investigador. Además, estos equipos son costosos por lo que requieren ser colocados en áreas seguras.

\section{Cazando Cocodrílidos}

Una ventaja de la caza de cocodrílidos es que se pueden seleccionar animales específicos. La mayoría de los cocodrílidos son capturados por este método. Los cocodrílidos más pequeños (menos de $1 \mathrm{~m}$ de largo total) suelen ser capturados con las manos, pinzas, lazos corredizos, pequeñas tripochetas cargadas con plomo, y distintos tipos de redes, incluyendo redes de inmersión, redes de lanzamiento y redes en tren. Los cocodrílidos de mayor tamaño son capturados mediante lazos corredizos más grandes (lazos corredizos de alambre que se cierren por sí solos son los mejores), pequeños arpones, ganchos triples pequeños y redes. 


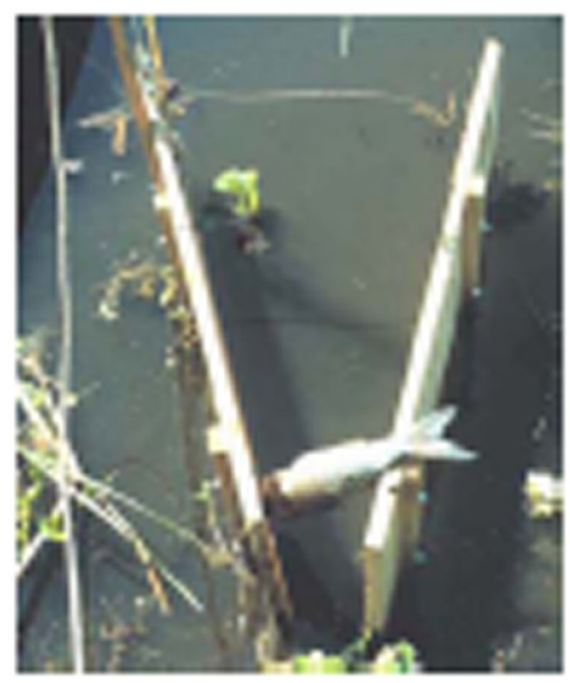

A. Trampa de Lazo Corredizo Cebada

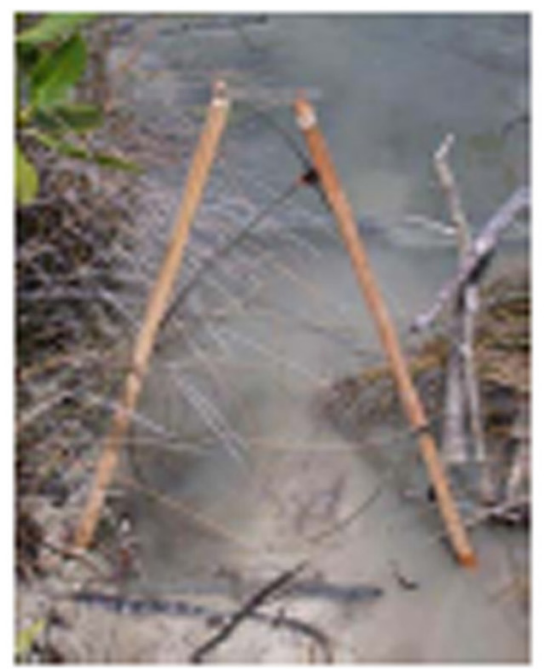

C. Trampa para Atrapar Animales Caminando

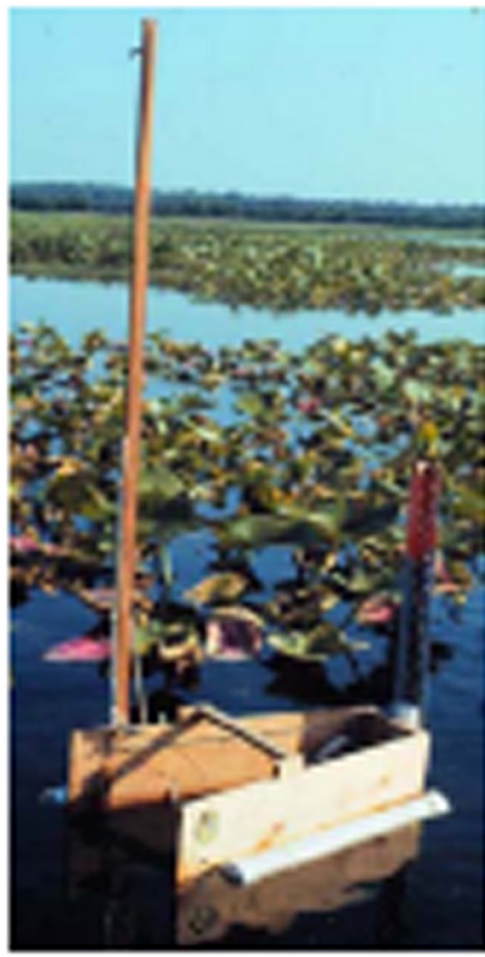

B. Trampa Flotante para Atrapar Animales Nadando

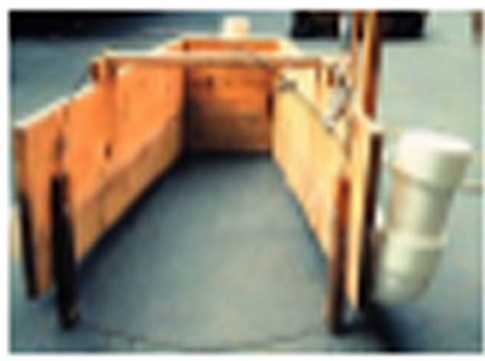

D. Acercamiento de la Trampa Flotante

Figura 4. Ejemplos de trampas de lazos corredizos para atrapar animales caminando y nadando. Créditos fotográficos: A. Phil Wilkinson, B. Graham Webb, C. Michael Cherkiss, y D. Graham Webb.

\section{Captura Manual/Pinzas}

Un investigador con experiencia puede agarrar un animal pequeño por detrás de la cabeza, y puede utilizar pinzas para extender el alcance de los brazos. Las pinzas Pilstrom son usualmente utilizadas para culebras, pero pueden atrapar animales de hasta 90 $\mathrm{cm}$. de largo. Las pinzas Pilstrom son una vara tubular de aluminio con unas pinzas adjuntas a uno de los extremos de la vara y un mecanismo para apretar en el otro extremo (Figura 5). El extremo con pinzas rodea y atrapa al cocodrílido por su cuello o cola para poder ser levantado y metido en un cubo o simplemente ser agarrado. Las manos y las pinzas no son efectivas para animales de más de $1.25 \mathrm{~m}$ de largo, que fácilmente pueden liberarse y morder la mano que lo está agarrando. Aún los cocodrílidos de $1 \mathrm{~m}$ de largo prueban el límite de la mayoría de las pinzas y deben ser extraídos rápidamente del agua a un bote o deben ser sujetados a un sustrato para prevenir la pérdida del animal o causar lesiones al mismo (Webb y Messel 1977). 


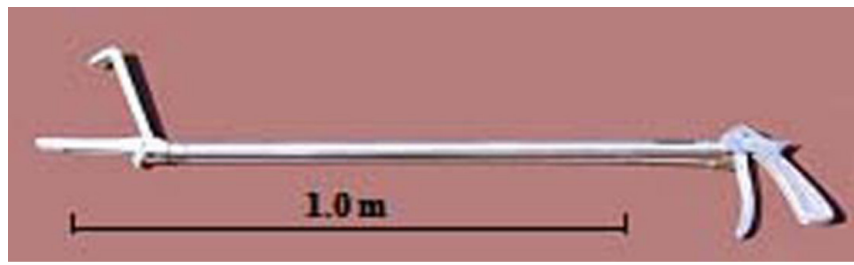

Figura 5. Pinzas Pilstrom para capturar cocodrilos pequeños. Créditos fotográficos: Michael Cherkiss.

\section{Redes}

Los cocodrílidos de menos de $1.2 \mathrm{~m}$ de largo total pueden ser capturados con redes de inmersión. Estas redes pueden construirse con la red alineada con la manija para recoger (Jones 1966), o formando un ángulo con ésta, con el fin de presionar hacia abajo sobre el cocodrílido desde arriba (Webb y Messel 1977). Las redes en tren y de lanzamiento son efectivas en aguas abiertas pero pueden enredarse con vegetación o rocas.

\section{Dardos}

Los dardos son arpones de cacería modificados (Jones 1966; Webb y Messel 1977; McDaniel y Hord 1990) donde el tamaño de la cabeza que penetra es reducido para que la profundidad de penetración en el animal sea minimizada. Esta modificación disminuye la probabilidad de lesionar al animal objetivo. Los dardos son adheridos a un extremo de una vara que se lanza manualmente. El dardo, amarrado a una soga, luego se separa de la vara (Figura 6). La fuerza y precisión juegan un rol crítico para lograr un lanzamiento exitoso. Los dardos deberían caer en áreas donde la piel sea relativamente suave y alejados de los órganos internos. Los mejores lugares para colocar los dardos son las mandíbulas y a los lados de la cola. Lograr colocar el dardo en el lugar adecuado desde un bote en movimiento puede ser una tarea difícil, particularmente si el animal se está zambullendo. El dardo tiene que lanzarse con suficiente fuerza para penetrar el cuero. Lanzamientos punzantes cortos representan la mejor combinación de fuerza y precisión. A pesar de que se ha logrado arponear a animales de tan solo $1 \mathrm{~m}$ de largo total, el área segura a apuntar es tan pequeña en aquéllos de menos de $1.5 \mathrm{~m}$ de largo total que el riesgo de lesionar al animal aumenta dramáticamente. Los dardos son utilizados principalmente para mantener una línea en el cocodrílido hasta que sea enlazado, ya que pueden zafarse fácilmente mediante movimientos de forcejeo. En situaciones de cacería, arpones más grandes de lanzamiento manual pueden ser utilizados, así como dispositivos propulsados. Dos métodos bastante utilizados para lanzar las cabezas de arpón son mediante el uso de arcos de caza y pistolas de lanzas. En situaciones de captura y liberación es muy difícil propulsar un dardo con suficiente precisión y fuerza como para penetrar el cuero sin causar lesiones.

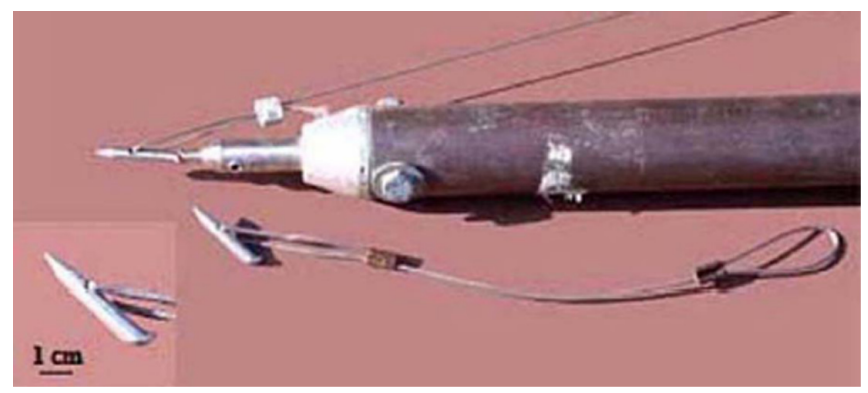

Figura 6. Dardo de Palanca utilizado para capturar cocodrílidos. Créditos fotográficos: Michael Cherkiss.

\section{Lazos Corredizos}

El uso de lazos corredizos para la captura de cocodrílidos es una técnica común y funciona muy bien cuando es posible acercarse al animal suficientemente como para colocar el lazo corredizo alrededor de su cuello (Chabreck 1963; Jones1966; Mazzotti 1983; McDaniel y Hord 1990). Es mejor preparar el lazo corredizo de modo que se rompa libremente de la vara previniendo un dispositivo poco manejable mientras el cocodrilo forcejea, y permitiendo el agotamiento del animal fuera del bote antes de ser montado a bordo. El lazo se ata a una línea de buena calidad la cual se une a un flotador o al bote de captura. Los flotadores deben ser utilizados al capturar cocodrílidos grandes en botes pequeños, para poder lanzar la estructura entera al agua en el caso de que el animal esté forcejeando y exista el riesgo de que logre voltear el bote. El animal puede ser recuperado más adelante. Los lazos corredizos pueden ser fijados halando o tirando bruscamente. Los investigadores aguantan la línea y, después de colocar el nudo corredizo alrededor del cuello del animal, halan la línea rápidamente, cerrando de este modo el nudo. El investigador halando logrará sacar de un tirón la vara de manera similar a como se coloca un anzuelo en la pesca. 
Las varas de captura pueden ser fabricadas a partir de madera, bambú, fibra de vidrio, plástico y otros materiales, dependiendo de la preferencia y de la disponibilidad. Las varas deben ser rígidas y ligeras; aquéllas usadas para halar de un tirón deben tener cierta flexibilidad. Las varas telescópicas pueden facilitar el transporte. Los nudos corredizos se unen a las varas con sujetadores de resorte o con cintas (Figura 7).

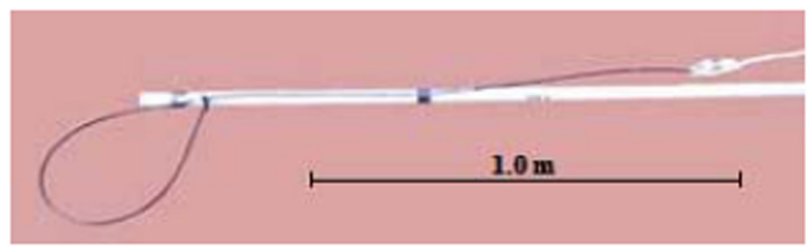

A. Lazo Corredizo

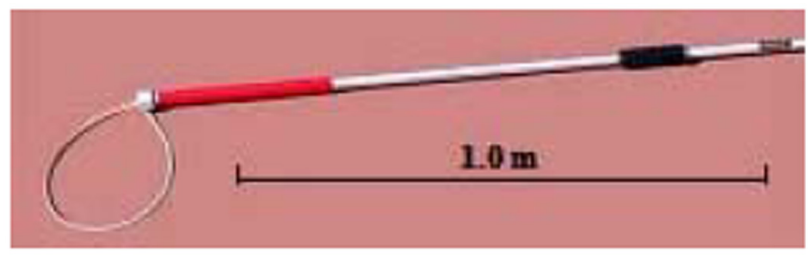

B. Tubo para Atrapar Perros

Figura 7. Lazo corredizo de alambre adjunto a un tubo de PVC y tubo para atrapar perros utilizados en la captura de cocodrílidos. Créditos fotográficos: Michael Cherkiss.

\section{Ganchos de Arrebatamiento}

Los ganchos triples se pueden lanzar más allá de un cocodrílido para atrapar al animal durante la recuperación (McDaniel y Hord 1990) (Figura 8). Los cocodrílidos en pozos que son inaccesibles pueden ser atrapados con los ganchos triples colgados de un helicóptero que maniobra. El colector luego salta al pozo y remolca al cocodrilo a la orilla más cercana para ser procesado. Las varas de pesca también pueden ser utilizadas para lanzar el gancho triple sobre la parte posterior del cocodrílido y engancharlo por la cola o por la parte posterior. Puesto que los ganchos pueden salirse causando lesiones cuando un cocodrilo forcejea, el animal debe ser amarrado tan pronto esté al alcance.

\section{Manipulando Cocodrílidos}

El tamaño de cocodrílidos que pueden ser montados en el bote depende del tamaño del bote que se esté utilizando y de la experiencia del equipo de

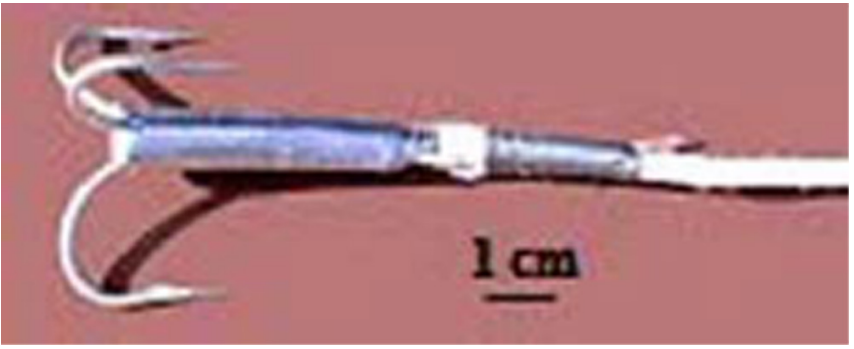

Figura 8. Gancho de lanzamiento y arrebatamiento utilizado en la captura de cocodrílidos. Créditos fotográficos: Michael Cherkiss.

captura. A cocodrílidos de $0.5 \mathrm{~m}$ de largo total o de mayor longitud se les debe cerrar el hocico asegurado antes de ser manipulados, para evitar que muerdan objetos duros y se rompan los dientes así como para prevenir que muerdan al investigador. Cuerdas, lazos corredizos (cuerda y alambre), ataduras plásticas de cable y ligaduras, pueden ser utilizados para cerrar el hocico; cintas de conducto o eléctricas pueden asegurar el hocico cerrado. Para asegurar los hocicos de animales más grandes se puede utilizar una tira de goma resistente con una línea atada a la misma. Después de la liberación, cuando el cocodrílido se encuentra a cierta distancia del capturador, el elástico puede ser removido halando la línea. Bajo ninguna circunstancia se debe permitir que un cocodrílido se escape o sea liberado con el hocico aún asegurado.

Una vez que el hocico ha sido cerrado, un cocodrílido puede ser asegurado aún más amarrando las patas dorsalmente (únicamente durante cortos períodos de tiempo), tapando sus ojos y oídos y, si es necesario, sujetando al animal a un tablero o a otro objeto duro que sea conveniente. No existen métodos de captura química de cocodrílidos que sean seguros; sin embargo, los animales pueden ser inmovilizados para la toma de medidas, para desplazamiento, y para cirugía (Loveridge y Blake 1987). El relajante muscular Flaxedil (triyoduro de gallamina) se ha utilizado con éxito en Crocodylus niloticus (Loveridge y Blake 1987; Leslie 1997) y en C. porosus (Walsh 1987). El antídoto para Flaxedil es neostigmina metilsulfato (Loveridge y Blake 1987). Es probable que la dosis de la droga y la conveniencia de su uso difieran entre especies. Una jeringa es el medio más simple y eficaz para suministrar una inyección intramuscular. El mayor riesgo de las drogas de inmovilización es 
la inyección accidental de la persona que las administra (Buys 1973). La preparación para el tratamiento de emergencia por inyección accidental debe formar parte de cualquier procedimiento de inmovilización.

Los animales que son capturados, procesados y liberados rápidamente muestran poco efecto perjudicial de la experiencia. Los cocodrílidos que han sido inmovilizados, o que han luchado en una trampa cualquiera sea la cantidad de tiempo, necesitan un período de recuperación. Loveridge y Blake (1987) enfatizaron en la importancia de colocar a los animales que han sido tratados con Flaxedil en aguas someras durante la recuperación para prevenir de esta manera un ahogamiento. A los animales que han sido manipulados después de ser capturados se les debe permitir recuperarse a su propio paso. Nunca se debe permitir que los cocodrílidos se calienten o enfríen en exceso durante la manipulación y recuperación de los mismos.

\section{Literatura Citada}

Bayliss, P. 1987. Survey methods and monitoring within crocodile management programmes. Pp. 157-175 En Webb, G. J. W., S. C. Manolis, y P. J. Whitehead (eds) Wildlife Management: Crocodiles and Alligators. Surrey Beatty and Sons, Chipping Norton, NSW.

Brandt, L. A., F. J. Mazzotti, J. R. Wilcox, P. D. Barker, G. C. Hasty y J. Wasilewski. 1995. Status of the American Crocodile (Crocodylus acutus) at a Power Plant Site in Florida, USA. Herpetological Natural History 3(1): 29-36.

Buys, A. C. 1973. Operator dangers associated with the use of immobilization drugs-accidents and emergency treatment. Pp. 77-83 En Young, E. (ed) The Capture and Care of Wild Animals. Human and Rousseau: Cape Town and Pretoria.

Chabreck, R H. 1963. Methods of capturing, marking, and sexing alligators. Proceedings of the Annual Conference of the Southeast Game and Fish Commission 17:47-50.

Graham, A., y R. Bell. 1969. Factors influencing the countability of animals. East African Agricultural and Forestry Journal 34 (Special Issue): 38-43.
Hutton, J. M., J. P. Loveridge y D. K. Blake. 1987. Capture methods for the Nile crocodile in Zimbabwe. Pp. 243-247 En Webb, G. J. W., S. C. Manolis, y P. J. Whitehead (eds) Wildlife Management: Crocodiles and Alligators. Surrey Beatty and Sons, Chipping Norton, NSW.

Jones, F. K. Jr. 1966. Techniques and methods used to capture and tag alligators in Florida. Proceedings of the Annual Conference of the Southeast Game and Fish Commission 19:98-101.

Kofron, C. P. 1989. A simple method for capturing large Nile crocodiles. African Journal of Ecology 27:183-189.

Kushlan, J.A. y F.J. Mazzotti. 1989a. Population Biology of the American Crocodile. J. Herpetol. 23(1): 7-21

Leslie, A. J. 1997. The ecology and physiology of the Nile crocodile, Crocodylus niloticus, in Lake St. Lucia, Kwazulu/Natal, South Africa. PhD. Dissertation: Drexel University, Philadelphia, PA.

Loveridge, J.P. y D.K. Blake. 1987. Crocodile immobilization and anaesthesia. Pp. 259-267 En Webb, G. J. W., S. C. Manolis, y P. J. Whitehead (eds) Wildlife Management: Crocodiles and Alligators. Surrey Beatty and Sons, Chipping Norton, NSW.

Magnusson, W. E., J. G. Caughley y G. C. Grigg. 1978. A double-survey estimate of population size from incomplete counts. Journal of Wildlife Management 42:174-176.

Magnusson, W.E., G.C. Grigg y J.A. Taylor. 1980. An Aerial Survey of Potential Nesting Areas of Crocodylus porosus on the West Coast of Cape York Peninsula. Australian Wildlife Resource 7: 465-78.

Magnusson, W. E. 1982. Techniques of surveying for crocodilians. Pp. En Crocodiles: Proceedings of the $5^{\text {th }}$ Annual Working Meeting of the Crocodile Specialist Group of the Species Survival Commission of IUCN-The World Conservation Union, Gland, Switzerland. 
Mazzotti, F.J. 1983. The ecology of the American crocodile in Florida. Ph.D. Thesis. Pennsylvania State University. University Park. PA

Mazzotti, F.J. y L.A. Brandt. 1988. A method for live trapping wary crocodilians. Herp. Rev. 10:40-41.

McDaniel, J., y L. Hord. 1990. Specialized equipment and techniques used in alligator management and research. Proceedings of the $10^{\text {th }}$ Working Meeting of the Crocodile Specialist Group of the Species Survival Commission of IUCN-The World Conservation Union 2:20-38.

McNease, L., N. Kinley, T. Joanen, D. Richard y D. Richard. 1994. Distribution and Relative Abundance of Alligator Nests in Louisiana Coastal Marshes. Proceedings of the $12^{\text {th }}$ Working Meeting of the Crocodile Specialist Group of the Species Survival Commission of IUCN- The World Conservation Union, vol 2. ISBN 2-8317-0239-9.

Murphy, T. M. Jr., y T. T. Fendley. 1975. A new technique for live trapping of nuisance alligators. Proceedings of the Annual Conference of the Southeast Game and Fish Commission 27:308-311.

OBrien, T. 1990. A comparison of 3 survey methods for estimating relative abundance of rare crocodilians. Proceedings of the $10^{\text {th }}$ Annual Working Meeting of the Crocodile Specialist Group of the Species Survival Commission of IUCN-The World Conservation Union 2:91-108.

Pacheco, L. F. 1996. Wariness of caiman populations and its effect on abundance estimates. Journal of Herpetology 30:123-126.

Petokas, F. J. y N. M. Alexander. 1979. A new trap for basking turtles. Herpetological Revue. 10:90.

Pitman, C. R. S. 1941. About crocodiles. The Uganda Journal. 9:89.

Thorbjarnarson, J. B. 1988. The Status and Ecology of the American Crocodile in Haiti. Bulletin of the Florida State Museum Biological Sciences 33 (1).
Thorbjarnarson, J. B. y G. Hernandez. 1988. Recent Investigations into the Status of Orinoco Crocodiles in Venezuela. Proceedings of the $9^{\text {th }}$ Working Meeting of the Crocodile Specialist Group of the Species Survival Commission of IUCN- The World Conservation Union, vol 2. ISBN 2-8317-0009-4.

Thorbjarnarson, J. B., R. R. Soberon, M A. Tabet, R R. Taragona y R. Da Silveira. 2000. On the use of camera traps to study crocodilian nesting behavior. Crocodile Specialist Group Newletter. 19 (3):17-18.

Waddle, J.H., K.G. Rice, y P.H. Franklin. 2003. From the Field- Using Personal Digital Assistants to Collect Wildlife Field Data. Wildlife Society Bulletin. 31 (1): 306-309.

Walsh, B. 1987. Crocodile capture methods used in the Northern Territory of Australia. Pp. 249-252 In Webb, G. J. W., S. C. Manolis, y P. J. Whitehead (eds) Wildlife Management: Crocodiles and Alligators. Surrey Beatty and Sons, Chipping Norton, NSW.

Webb, G. J. W., y H. Messel. 1977. Crocodile capture techniques. Journal of Wildlife Management 41:572-575.

Webb, G. J. W., y H. Messel. 1979. Wariness in Crocodylus porosus. Australian Wildlife Research 6:227-234.

Wilkinson, P. M. 1994. A walk-through snare design for the live capture of alligators. Proceedings of the $12^{\text {th }}$ Working Meeting of the Crocodile Specialist Group of the Species Survival Commission of IUCN-The World Conservation Union 2:74-76.

Woodward, A. R., y W. R. Marion. 1979. An evaluation of factors affecting night-light counts of alligators. Proceedings of the Annual Conference of the Southeast Game and Fish Commission 32:291-302. 